\title{
Differential activity of methylene blue against erythrocytic and hepatic stages of Plasmodium
}

\author{
Henriette Bosson-Vanga ${ }^{1,2^{*}} \mathbb{0}$, Jean-François Franetich ${ }^{1}$, Valérie Soulard ${ }^{1}$, Daniel Sossau ${ }^{1,3}$, Maurel Tefit ${ }^{1}$, \\ Bocar Kane ${ }^{4}$, Jean-Christophe Vaillant ${ }^{5}$, Steffen Borrmann ${ }^{6,7}$, Olaf Müller ${ }^{8}$, Nathalie Dereuddre-Bosquet ${ }^{9}$, \\ Roger Le Grand ${ }^{9}$, Olivier Silvie ${ }^{1}$ and Dominique Mazier ${ }^{1,10^{*}}$
}

\begin{abstract}
Background: In the context of malaria elimination/eradication, drugs that are effective against the different developmental stages of the parasite are highly desirable. The oldest synthetic anti-malarial drug, the thiazine dye methylene blue (MB), is known for its activity against Plasmodium blood stages, including gametocytes. The aim of the present study was to investigate a possible effect of MB against malaria parasite liver stages.

Methods: MB activity was investigated using both in vitro and in vivo models. In vitro assays consisted of testing MB activity on Plasmodium falciparum, Plasmodium cynomolgi and Plasmodium yoelii parasites in human, simian or murine primary hepatocytes, respectively. $M B$ in vivo activity was evaluated using intravital imaging in BALB/C mice infected with a transgenic bioluminescent $P$. yoelii parasite line. The transmission-blocking activity of MB was also addressed using mosquitoes fed on MB-treated mice.

Results: MB shows no activity on Plasmodium liver stages, including hypnozoites, in vitro in primary hepatocytes. In BALB/c mice, MB has moderate effect on P. yoelii hepatic development but is highly effective against blood stage growth. $M B$ is active against gametocytes and abrogates parasite transmission from mice to mosquitoes.
\end{abstract}

Conclusion: While confirming activity of $\mathrm{MB}$ against both sexual and asexual blood stages, the results indicate that MB has only little activity on the development of the hepatic stages of malaria parasites.

Keywords: Malaria, Methylene blue (MB), Exoerythrocytic stages, Plasmodium, Blocking malaria transmission

\section{Background}

Malaria remains a major cause of morbidity and mortality in many regions of the world [1]. In 2016 the World Health Organization estimated a total of 212 million cases of malaria, which resulted in 429,000 deaths. African populations, particularly children younger than 5 years old, are the most affected by this disease [2].

\footnotetext{
*Correspondence: bossonhenriette@gmail.com; dominique. mazier@upmc.fr

1 Sorbonne Université, Inserm, CNRS, Centre d'Immunologie et des Maladies Infectieuses, U1135, ERL8255, CIMI-Paris, F-75013 PARIS, France

${ }^{10}$ Service de Parasitologie-Mycologie, Centre National de Référence du Paludisme, AP-HP, Groupe Hospitalier Pitié Salpêtrière, 83 Bd de l'hôpital, 75013 PARIS, France

Full list of author information is available at the end of the article
}

Human malaria is caused by five different species of Plasmodium parasites. Plasmodium falciparum and Plasmodium vivax are the most common forms with P. falciparum being the deadliest. Due to the ability to rapidly develop drug resistance, Plasmodium parasites continue to be a major challenge for effective case management $[3,4]$. Malaria parasites consist of several life cycle stages that have to be individually targeted to reach malaria elimination [5].

Most of the drugs used for the treatment of malaria act as erythrocytic stage inhibitors. The ambitious goal of malaria elimination, however, requires strategies to prevent parasite transmission between the human host and the mosquito vector, thus targeting the hepatic or the gametocyte developmental stages [6]. To date, 
primaquine $(\mathrm{PQ})$ is the only approved compound active against all stages of Plasmodium development: liver stages, including hypnozoites, and blood stages, including gametocytes, thereby also blocking parasite transmission to mosquitoes. However, PQ causes haemolytic anaemia in moderate to severely glucose-6-phosphate dehydrogenase (G6PD)-deficient individuals [7]. Moreover, recent studies show that PQ is ineffective in people with low metabolizing cytochrome P450 2D6 genotypes [8]. Attempts to develop a replacement for PQ led to tafenoquine, a drug of the same class with partly more favourable pharmacokinetics, but which is still deleterious in patients with G6PD deficiency $[9,10]$. PQ was initially derived from methylene blue (MB) $[11,12]$, the first synthetic drug ever used against malaria [13]. MB is a subversive substrate and specific inhibitor of the $P$. falciparum disulfide reductase. It played a major role in malaria treatment before and during World War II [14]. In recent years, there was a surge of interest in $\mathrm{MB}$ as an anti-malarial agent when $P$. falciparum glutathione reductase was identified as a new drug target [15]. In vitro $\mathrm{MB}$ is active against parasites resistant to standard drugs [16], and oral MB was used to treat uncomplicated malaria in Burkina Faso [17]. A triple combination of $\mathrm{MB}$ with artesunate and amodiaquine was shown to be effective against the gametocytes of $P$. falciparum [18]. Furthermore, these clinical trials in Africa showed that effective antiplasmodial doses of $\mathrm{MB}$ are safe in both adults and children including G6PD-deficient individuals [19]. MB appears to be a potentially useful partner drug for existing artemisinin-based combination therapy (ACT), which is first-line anti-malarial treatment. On the contrary to its well-described inhibitory activity on blood stages, whether MB is able to interfere with Plasmodium liver stages is still an open question. Here, MB's activity was assessed against Plasmodium spp liver stages, including hypnozoites, in vitro and in vivo, and its transmission blocking activity was analysed in vivo.

\section{Methods}

\section{Drugs and chemicals}

The chlorure of methylthioninium Proveblue was used in this study. It is a MB preparation provided by Provepharm; a company specialized in the development of pharmaceutical products. Today, Proveblue ${ }^{\circledR}$ methylene blue active pharmaceutical ingredient (API) is fully compliant with most recent pharmacopoeial standards on MB. Proveblue was obtained from Olaf Müller. Figure 1 shows the chemical structure of the compound. For in vitro and in vivo assays $\mathrm{MB}$ was dissolved in distilled water. Primaquine used in these studies was obtained from Sigma-Aldrich, USA. Stock solutions were prepared at $10 \mathrm{mg} / \mathrm{ml}$ in distilled water. D-Luciferin, provided by

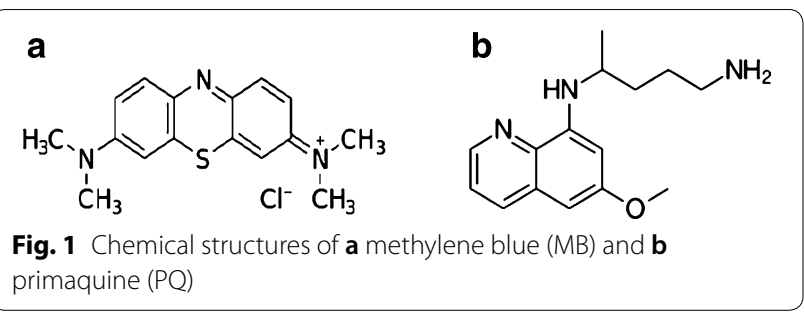

OZBioscences, a substrate for luciferase was dissolved in $1 \times$ phosphate-buffered saline (PBS) (Gibco) and injected intraperitoneally at $100 \mathrm{mg} / \mathrm{kg}$.

\section{Parasites and sporozoites isolation}

Three species of Plasmodium were used in this study:

1. Plasmodium falciparum (NF54 strain) sporozoites were obtained from infected salivary glands of Anopheles stephensi 14-21 days after an infective blood meal (Department of Medical Microbiology, Radboud University Medical Center, Nijmegen, The Netherlands).

2. Plasmodium cynomolgi (M strain) sporozoites were obtained from infected salivary glands of An. stephensi 14-16 days after an infective blood meal (blood infected with $P$. cynomolgi was obtained from Macaca fascicularis, the natural host of P. cynomolgi, infected with cryopreserved blood stages) (CEA, Fontenay aux Roses, France).

3. Plasmodium yoelii (17XNL strain), line expressing GFP-Luciferase (GFP-luc) was generated using a 'Gene Out Marker Out' strategy [20].

Infected salivary glands were aseptically removed by hand dissection, crushed in a potter and filtered through a $40-\mu \mathrm{m}$ filter for sporozoite isolation (Cell Strainer, BD BioSciences, USA). Sporozoites were counted using a disposable Glasstic microscope slide (KOVA, USA).

\section{Mice and mosquitoes}

Six to 8 weeks old female BALB/c mice (18-22 g), obtained from Janvier (Le Genest-Saint-Isle, France) were used. For in vivo transmission blocking studies with $P$. yoelii, 4-5 days old An. stephensi female mosquitoes were used as experimental vectors.

\section{Hepatocytes}

Primary human hepatocytes were isolated from liver segments obtained from adult patients undergoing partial hepatectomy (Service de Chirurgie Digestive, hépato-bilio pancréatique, Hôpital Pitié Salpêtrière, Paris France). Primary simian hepatocytes were isolated from liver 
segments collected from healthy Macaca fascicularis from CEA, Fontenay aux Roses, France or ICM, Hôpital Pitié Salpêtrière, Paris France. Primary murine hepatocytes were isolated from liver segments collected from BALB/c mouse.

All hepatocytes were isolated using collagenase perfusion as previously described [21,22]. Simian hepatocytes were immediately cryopreserved with a Nicool-Freezal (Air liquide Santé, Marne la vallée) and then used when needed after fast thawing at $37{ }^{\circ} \mathrm{C}$. Human and murine hepatocytes were used in fresh cultures. Cells were seeded in 96-well plates (Falcon by Becton-Dickinson Labware Europe, France) coated with collagen I (BD Biosience, USA), at a density of 80,000 cells per well. Human, simian and murine hepatocytes were maintained at $37{ }^{\circ} \mathrm{C}$ in $5 \% \mathrm{CO}_{2}$ in William medium (Gibco) supplemented with $10 \%$ of fetal bovine serum (FBS, FetalClone, Hyclone), 1\% penicillin-streptomycin (Gibco), $5 \times 10^{-3} \mathrm{~g} / \mathrm{l}$ insulin (Sigma Aldrich, USA) and $5 \times 10^{-5}$ hydrocortisone (Upjohn Laboratories SERB, France) until infection with sporozoites. For human hepatocytes, the complete medium was supplemented with $2 \%$ dimethyl sulfoxide (DMSO, Sigma- Aldrich, USA) until infection.

\section{Assessment of liver stage development in vitro}

Hepatocytes were seeded into collagen-coated black 96-wells plates and maintained at $37{ }^{\circ} \mathrm{C}$ in $5 \% \mathrm{CO}_{2}$ in a complete medium. On the day of infection, $3 \times 10^{4}$ sporozoites of $P$. falciparum, $P$. cynomolgi or $P$. yoelii resuspended in complete medium were added to the respective host cell cultures (human, simian or murine). The infected culture plates were centrifuged for $10 \mathrm{~min}$ at $900 \mathrm{~g}$ allowing fast parasite sedimentation and further incubated for $3 \mathrm{~h}$ at $37{ }^{\circ} \mathrm{C} / 5 \% \mathrm{CO}_{2}$ allowing parasites invasion into the hepatocytes. Serial dilution of the tested drugs, $\mathrm{MB}$ and $\mathrm{PQ}$ were added to the cultures. Culture medium containing the appropriate drug concentration was changed daily and cells were fixed $48 \mathrm{~h}$ post infection (pi) for P. yoelii, 6 days pi for P. falciparum or 8 days pi for P. cynomolgi.

\section{Parasite counting and schizont size determination using high-content imaging}

The number, size and shape of the parasites were determined using a CellInsight High Content Screening platform equipped with the Studio HCS software (ThermoFisher Scientific). Parasite immunostaining was done using a $\alpha$-Plasmodium HSP70 antibody raised in mice and a secondary anti-mouse antibody coupled to AlexaFluor 488 [23]. Host cell and parasite nuclei were labelled with 4',6-diamidino-2-phenylindole (DAPI). Thirty-seven images, representing more than $95 \%$ of the total bottom surface area of a culture well in 96-well plates, were captured for analysis. The custom script used for counting and measuring parasites first required identification of the objects on the basis of a fluorescence intensity threshold. The object identity was then further validated based on size and morphological (shape) criteria. The presence of DAPI-associated fluorescence in the selected objects allowed for their final selection and rejection of false positives. Inhibitory concentration 50 $\left(\mathrm{IC}_{50}\right)$ value is the drug concentration at which a $50 \%$ reduction of the exo-erythrocytic forms (EEFs) number was observed, as compared to the control cultures. $\mathrm{IC}_{50}$ were calculated using ICEstimator software version 1.2 [24].

The parasite size reduction is calculated on the average object area using the total surface area of each selected object $\left(\mu \mathrm{m}^{2}\right)$. Cell confluence for primary hepatocytes was measured by counting the absolute number of host cell nuclei using a script that first requires identification of the objects on the basis of the DAPI fluorescence intensity threshold and then involved a validation step based on size and morphological (shape) criteria. The objects on the edge of the pictures were excluded. The toxic concentration $50\left(\mathrm{TC}_{50}\right)$ value was determined as the drug concentration at which a $50 \%$ reduction of the cell confluence was estimated. With $\mathrm{IC}_{50}$ and $\mathrm{TC}_{50}$, therapeutic index (TI) was calculated $\left(\mathrm{TC}_{50} / \mathrm{IC}_{50}\right)$.

\section{Assessment of liver stage in vivo development}

Five mice per treatment group were used. Experimental groups were treated with $\mathrm{MB}$ at 50 and $100 \mathrm{mg} / \mathrm{kg}$ by intra-peritoneal (ip) injection. Untreated infected mice, non-infected mice (infection controls) as well as PQ-treated $(50 \mathrm{mg} / \mathrm{kg})$ mice (control drug) were also included. The drugs were administrated on days $-1,0$, +1 , and mice were challenged on day 0 by retro-orbital injection of $P$. yoelii (GFP-luc strain) sporozoites $(10,000$ per mouse). In vivo imaging was performed $44 \mathrm{~h}$ postinfection to assess liver stage development. When parasites were detected in the liver, treatment was continued until day 5 to assess blood stage activity. On day 4 and 6 pi, in vivo imaging and GIEMSA-stained blood smears were performed to monitor blood stage development using methods described previously [25]. IVIS Spectrum (Caliper Life Science, Hanover, MD, USA) was used to measure luciferase activity. Prior to analysis the mice were injected ip with D-luciferin $(100 \mathrm{mg} / \mathrm{kg})$, anesthetized with isoflurane and imaged $10 \mathrm{~min}$ post-injection. Images were analysed using the living Image 3.0 software (Capiler Life Science, Hanover, MD, USA). For blood stage assessment, luminescence (total flux photons/seconde) and Giemsa-stained blood smears were done on 
days 3, 4 and 6 pi to evaluate the blood stage prepatent period in mice after sporozoite inoculation.

\section{In vivo blood stage and transmission blocking activity assays}

Mice were infected by ip inoculation of $10^{7}$ erythrocytes parasitized with GFP-luc P. yoelii and separated in two groups. The first group was treated from day 0 to day 3 . For the second group, treatment was initiated 4 days after the infection, $2 \mathrm{~h}$ before mosquito feeding [26]. For each compound (Control: PBS, MB $50 \mathrm{mg} / \mathrm{kg}$ ) and for each treatment protocol, mice were randomly separated into groups of five animals. At day 3, the presence of gametocytes was checked by microscopic analysis of Giemsastained blood films and their functionality was assessed using microgamete exflagellation assay. Mice were anesthetized and placed according to the treatment on the top of individual cages containing 100 glucose-starved An. stephensi female mosquitoes, which were allowed to feed for $1 \mathrm{~h}$. Unfed mosquitoes were removed from the cage. Seven days after the blood meal, 30-40 mosquitoes were dissected and their midguts examined under light microscope $(400 \times)$ to assess oocyst presence. Two weeks after the blood meal, salivary glands of 30-40 mosquitoes per group were dissected to determine the average number of sporozoites per mosquito in each group. Inhibition activity was determined by determining the percentage of oocyste-positive mosquitoes (infection rate), the mean number of oocysts per mosquito (oocyst burden) and the mean number of sporozoites per mosquito.

\section{Statistical analysis}

Excel 2007 spreadsheet (Microsoft office) and GraphPad Prism 6 statistical Software (GraphPad. Software, San Diego, CA, USA) were used for data analysis. All values were expressed as means and standard deviations (SD). A $p$ value of 0.05 or less was considered as statistically significant.

\section{Results}

\section{In vitro activity against Plasmodium liver stages}

$\mathrm{MB}$ was tested at concentrations ranging from 0.02 to $100 \mu \mathrm{M}$ on primary murine, human and simian hepatocytes infected, respectively, with $P$. yoelii, $P$. falciparum and P. cynomolgi. MB and PQ (Fig. 1) cytotoxicity was evaluated by assessing cell confluence and number of DAPI-positive hepatocytes after drug treatment and antimalarial activity was evaluated by counting EEFs (Figs. 2 and 3 ).

As shown in Table $1, \mathrm{MB}$ had no effect $\left(\mathrm{IC}_{50}>100 \mu \mathrm{M}\right)$ on $P$. falciparum, P. cynomolgi and $P$. yoelii liver stages including P. cynomolgi hypnozoites (Fig. 2c). The corresponding $\mathrm{TI}\left(\mathrm{TI}=\mathrm{TC}_{50} / \mathrm{IC}_{50}\right)$ in these primary hepatocytes $\left(\mathrm{TC}_{50}>100 \mu \mathrm{M}\right)$ was very low $(\mathrm{TI}=1)$. As expected, PQ was highly active on $P$. falciparum and $P$. cynomolgi liver stages with $\mathrm{IC}_{50}$ of $0.6 \mu \mathrm{M}$ in human cells and $2.72 \mu \mathrm{M}$ in simian cells. The TI of PQ was, respectively, 66.5 in human hepatocytes $\left(\mathrm{TC}_{50} 39.93 \pm 2.29 \mu \mathrm{M}\right)$ and 14.22 in simian hepatocytes $\left(\mathrm{TC}_{50} 38.7 \pm 2.98 \mu \mathrm{M}\right)$. In addition, $\mathrm{MB}$ had limited effect on parasite development, as evaluated by measuring parasite size (Table 1 ). At $11 \mu \mathrm{M}, \mathrm{MB}$ induced a $10 \%$ reduction of $P$. falciparum and $P$. cynomolgi parasite area.

\section{In vivo activity against Plasmodium yoelii liver stage}

Two groups of BALB/c mice were treated with $\mathrm{MB}$ at 50 and $100 \mathrm{mg} / \mathrm{kg}$ or PQ at a concentration of $50 \mathrm{mg} / \mathrm{kg}$ one day prior the infection, the day of infection and 1 day post-infection. PQ $(50 \mathrm{mg} / \mathrm{kg})$ served as a positive control. The results illustrate that $\mathrm{MB}$ was unable to prevent parasite blood stage development (Fig. 4a). Mice treated with PQ were free of parasites throughout the infection experiment (Fig. 4a). Mean luminescence (photons/ sec) values collected from liver location of mice were $2.11 \times 10^{7}\left( \pm 6.39 \times 10^{6}\right)$ for untreated control group, $1.66 \times 10^{7}\left( \pm 3.07 \times 10^{6}\right)$ for MB $50 \mathrm{mg} / \mathrm{kg}$ and $2.97 \times 10^{6}$ $\left( \pm 2.00 \times 10^{6}\right)$ for MB $100 \mathrm{mg} / \mathrm{kg}$ at $44 \mathrm{~h} \mathrm{pi} \mathrm{(Fig.} \mathrm{4b).} \mathrm{Plas-}$ modium yoelii liver stage inhibition was, respectively, 21.43 and $85.9 \%$ for $\mathrm{MB}$ at 50 and $100 \mathrm{mg} / \mathrm{kg}$. At days 3 and 4 post-infection, the luminescence signal spread in the entire body due to parasite dissemination in the blood circulation. When the growth of parasites is compared between day 2 and day 4, the signal increased five times in the control group whereas in the groups treated with $\mathrm{MB}$ there was a reduction of the luminescence signal (Fig. 4b). In order to validate these results, mean luminescence was correlated with blood parasitaemia based on blood smears microscopic examination on days 3, 4 and 6 pi. Compared to untreated controls, blood parasitaemia was strongly diminished after treatment of mice on days 4 and 6 with 50 and $100 \mathrm{mg} / \mathrm{kg} \mathrm{MB}$. The protocol using $100 \mathrm{mg} / \mathrm{kg}$ showed a slightly increased effectiveness (Fig. 4c). These results show that MB has a moderate effect on liver stages, albeit at a level unable to prevent blood stage development. Conversely, MB strongly inhibits blood stage growth in vivo.

\section{In vivo transmission blocking assays}

In order to analyse the ability of $\mathrm{MB}$ to inhibit parasite transmission to mosquitoes, An. stephensi mosquitoes were fed on $P$. yoelii-infected $\mathrm{BALB} / \mathrm{c}$ mice with or without $\mathrm{MB}$ treatment. The mean number of oocysts per mosquito (oocyst burden) and the mean number of sporozoites per mosquito were dramatically decreased showing that MB efficiently reduces transmission (Fig. 5). Table 2 shows the mean parasitaemia and 


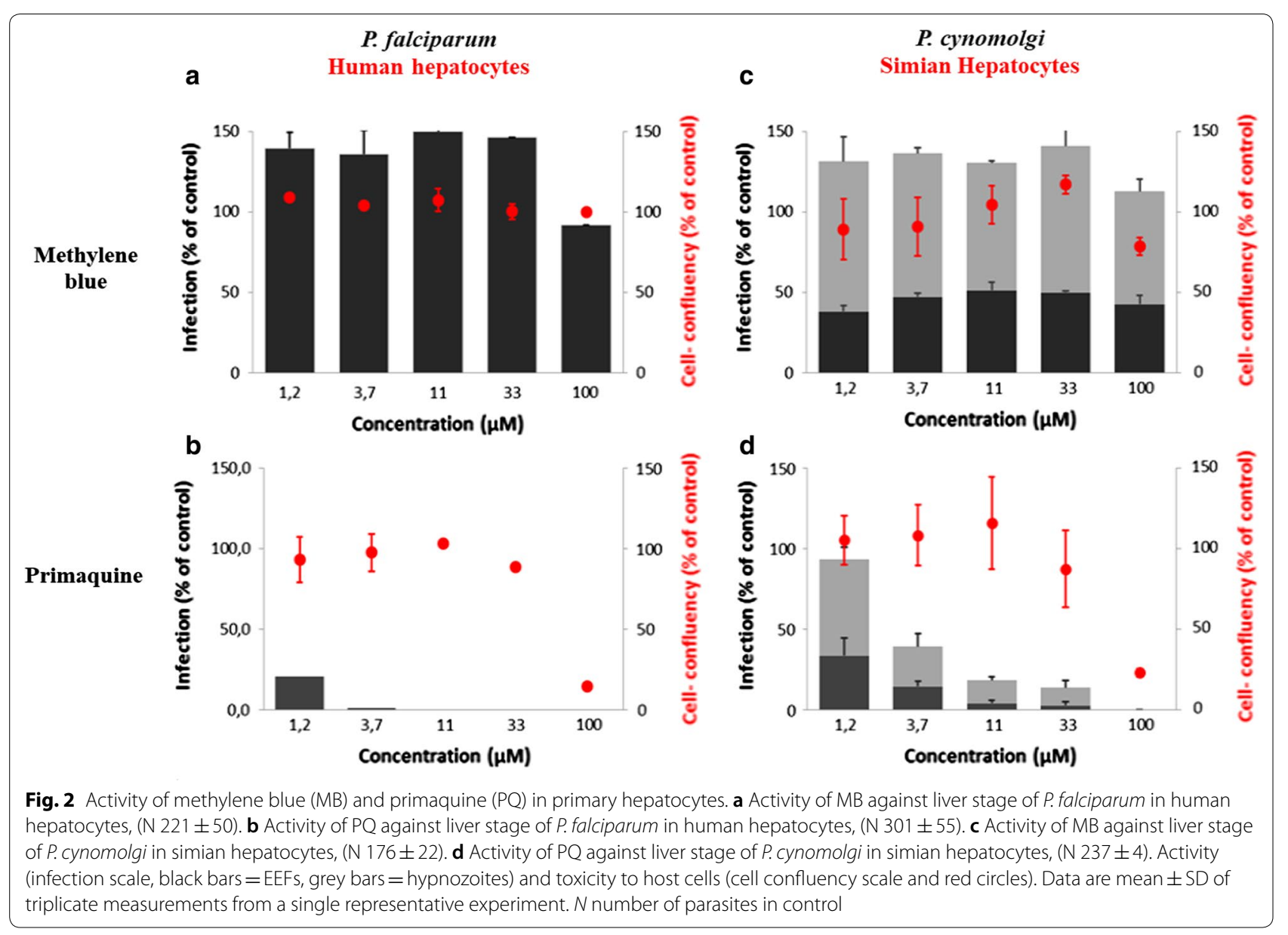

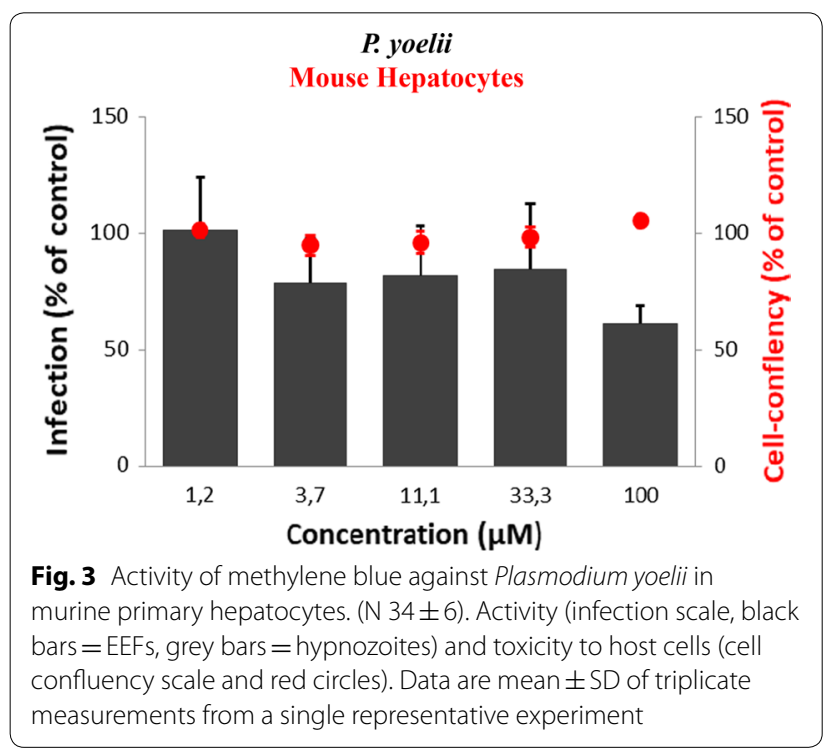

gametocytaemia before a mosquito's blood meal. Mice treated from day 0 to day 3 showed a remarkable reduction of parasitaemia of nearly $79 \%$ as well as a reduction of gametocytaemia of $100 \%$ compared to the control group. These results clearly show that MB can inhibit parasite transmission by eliminating gametocytes. Treatment applied $2 \mathrm{~h}$ before mosquito feeding had no effect on the gametocyte rate in the mice, as observed on blood smears, but induced a drastic reduction of both the mosquito infection rate and the number of oocysts in infected mosquito midguts dissected 1 week post infection (mean number of 128 oocysts per infected mosquito in the control group compared to 8 oocysts in the treated group). As expected, this decrease in the oocyst number led to a strong reduction in sporozoite numbers in salivary glands 2 weeks post mosquito blood meal (Fig. 5b). From this last experiment, the conclusion was that a shortterm exposure to $\mathrm{MB}$ is sufficient to prevent Plasmodium transmission to An. stephensi mosquitoes.

\section{Discussion}

Most currently available anti-malarial drugs primarily target the disease-causing parasite stages in the human blood system. However, to eradicate malaria, new drugs that block transmission of the parasite between the 
Table 1 Comparative inhibitory activities of Proveblue and primaquine on Plasmodium yoelii, Plasmodium falciparum and Plasmodium cynomolgi

\begin{tabular}{|c|c|c|c|c|c|c|}
\hline \multirow{2}{*}{$\begin{array}{l}\text { Drugs } \\
\text { Parasites }\end{array}$} & \multicolumn{3}{|c|}{ Methylene blue } & \multicolumn{3}{|l|}{ Primaquine } \\
\hline & $\mathrm{IC}_{50}(\mu \mathrm{M})$ & $\mathrm{TI}$ & $\begin{array}{l}\text { Size reduction at } 11 \mu \mathrm{M} \\
\text { (\%) }\end{array}$ & $\mathrm{IC}_{50}(\mu \mathrm{M})$ & $\mathrm{TI}$ & $\begin{array}{l}\text { Size } \\
\text { reduction } \\
\text { at } 11 \mu \mathrm{M}(\%)\end{array}$ \\
\hline P.yoelii (MH) & $>100$ & 1 & 10 & $0.64 \pm 0.1^{\mathrm{a}}$ & $110^{\mathrm{a}}$ & ND \\
\hline P. falciparum $(\mathrm{HH})$ & $>100$ & 1 & 10 & $0.6 \pm 0.06$ & 66.5 & 100 \\
\hline P. cynomolgi (SH) & $>100$ & 1 & 10 & $2.72 \pm 0.34$ & 14.22 & 70 \\
\hline
\end{tabular}

$\mathrm{MH}$ mouse hepatocytes; $\mathrm{HH}$ human hepatocytes; $\mathrm{SH}$ simian hepatocytes; $\mathrm{TI}$ therapeutic index: $\mathrm{TC}_{50} / \mathrm{IC}_{50}, N D$ not determined

a $[51]$

human host and the mosquito vector, and eliminate the various stages of the parasite during its cycle in the human body, are needed. It is surprising that few studies [27], to date, have examined drug activity against liver, sexual blood and mosquito stages of the malaria parasite at the same time. In this study, PVB activity was assessed on these different parasite stages. PVB is a MB preparation that complies with the European Pharmacopoeia, contains limited organic impurities and heavy metals of recognized toxicity, and has previously been demonstrated to possess anti-malarial activity on asexual and sexual blood stages. In vitro experiments confirmed PVB anti-malarial potency against the blood stage of $23 P$. falciparum strains that were resistant to various anti-malarial drugs [16]. In combination with mefloquine, quinine and dihydroartemisinin, PVB showed synergistic effects in vitro against 9 well-established $P$. falciparum strains $[28,29]$. Ex vivo experiments on 19 samples isolated from patients showed $\mathrm{IC}_{50}$ for PVB ranging from 0.88 to $40.2 \mathrm{nM}$ with a mean of $5.3 \mathrm{nM}$ [30]. PVB treatment at $1-10 \mathrm{mg} / \mathrm{kg}$ for 5 days significantly reduced or prevented cerebral malaria in mice [31-33]. In humans, several clinical trials conducted in Burkina Faso showed that oral MB was safe and effective in the treatment of uncomplicated falciparum malaria when combined with other antimalarials $[17,18,34-36]$. Another study reported moderate curative activity with $\mathrm{MB}$ monotherapy, illustrating the need for this slow-acting drug to be combined with fast-acting anti-malarials [37]. Another advantage of MB is that it has gametocytocidal properties and can reduce the transmission of $P$. falciparum. In fact, MB interferes in vitro on gametocyte development at all stages and can block transmission through clearance of stage $\mathrm{V}$ gametocytes [38]. Previous clinical observations showed that MB reduced gametocyte carriage rate in treated patients $[18,39]$. So far no published study has analysed the activity of MB on the hepatic stage of Plasmodium parasites. Here, several established models of malaria liver stages were used to evaluate the effectiveness of PVB. The $P$. yoelii murine model has two main interests, it allows: (1) a quick and 'easy' in vitro screening of the molecules; and, (2) to draw a parallel between in vitro and in vivo experiments. Murine Plasmodium species are commonly used to study the liver stage of infection in mice and in vitro, yet it is not clear how well these models reflect $P$. falciparum infections in humans. Then, two in vitro models: $P$. falciparum and $P$. cynomolgi were used to validate the results with $P$. yoelii model. The $P$. falciparum experiments provide direct data on pharmacodynamic effects against the liver and blood stages of this most important human malaria parasite. The $P$. cynomolgi system is being used as a model for $P$. vivax and replicates its capability to produce hypnozoites. This $P$. cynomolgi model has been used for decades to identify radical curative compounds against $P$. vivax $[40,41]$. In all these models, PVB showed no significant activity on Plasmodium liver stage infection in vitro. Interestingly, MB could partially reduce the parasite burden in the liver of $P$. yoeliiinfected mice; however this effect was observed only at the highest doses and was not sufficient to prevent transition to a blood stage infection. To date, there is only a limited number of therapeutics capable of eliminating Plasmodium liver stages [42, 43]. Whereas PQ, as well as tafenoquine, and atovaquone actively eliminate dividing Plasmodium liver stages, only PQ has a pronounced effect on the hypnozoites [22]. However, because of its potential to cause life-threatening acute intravascular haemolysis in individuals with severe G6PD deficiency, PQ cannot be widely used [44]. Thus, the development of new compounds with hypnozoitocidal activity suitable for mass administration has become a priority in the current drive to eliminate malaria.

In the present study, MB shows low or no activity in vitro in primary hepatocytes while $P Q$ is very effective. Next, in vivo experiments were performed to evaluate the effect of $\mathrm{MB}$ on hepatic and blood stages development. In vivo imaging of Plasmodium growth was used to measure the specific inhibition of parasite hepatic 


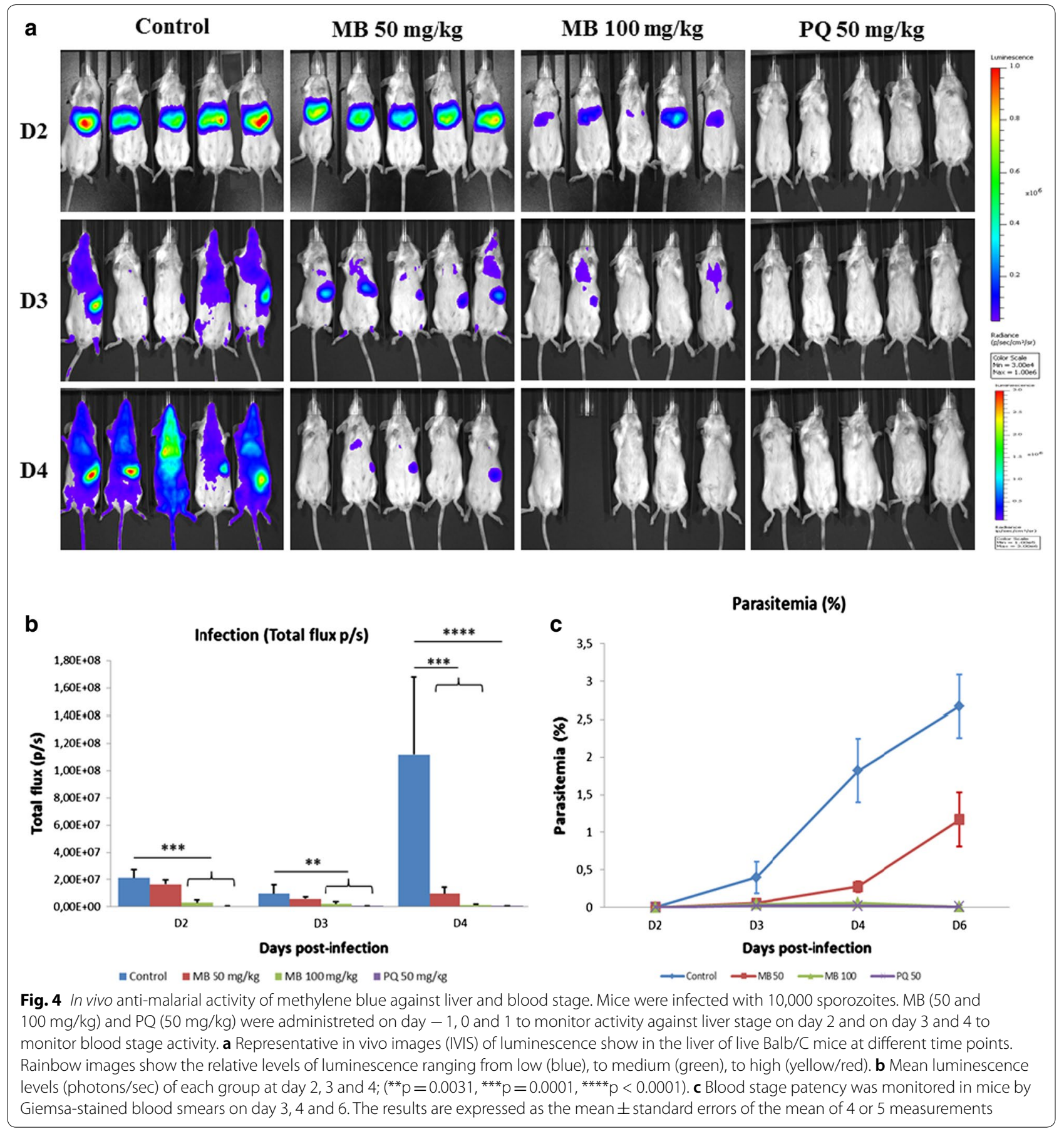

stage development and its consequences on blood stage spreading $[45,46]$. All control mice used showed a strong luminescence signal at $44 \mathrm{~h}$, as well as on day 3 and 4 post-infection while luminescence signal was abrogated in animals treated with PQ $(50 \mathrm{mg} / \mathrm{kg})$. In these experiments, while $\mathrm{MB}$ was not able to prevent spreading of the parasites into the central circulation (Fig. 4), an inhibitory effect was observed using the high dose of $100 \mathrm{mg} /$ $\mathrm{kg}$, demonstrating once again that to prevent erythrocytic infection, hepatic inhibition has to be close to $100 \%$. These results have to be considered with those obtained by Garavito in his thesis [47]. Studying the pharmacological effects of $M B$, he observed a slight delay in the appearance of the erythrocytic infection.

Finally, the activity of $\mathrm{MB}$ against $P$. yoelii blood stages was confirmed and its transmission blocking 

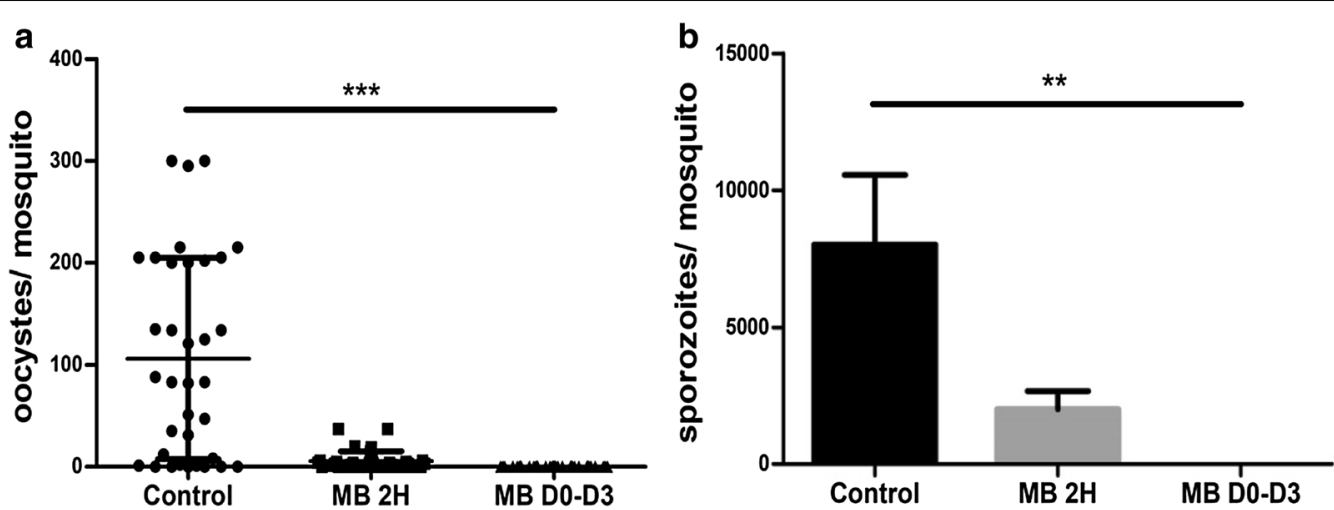

Fig. 5 Impact of methylene blue $50 \mathrm{mg} / \mathrm{kg}$ on sporogonic development. a Number of oocysts per midgut. Each point represents the oocyst number from an individual mosquito. Oocysts density in MB groups significantly different with the untreated group $\left(^{* * *} p<0.0001\right)$. b Average of sporozoites extracted from salivary glands of mosquitoes (Total of sporozoites/number of mosquitoes). $(* * p<0.0016)$

Table 2 Parasitaemia and gametocytaemia before feeding and prevalence of infected mosquitoes in each group

\begin{tabular}{lll}
\hline & Untreated & MB 2H \\
\hline Parasitaemia (\%) & $0.95 \pm 0.39$ & $1.36 \pm 0.18$ \\
Gametocytaemia (\%) & $0.08 \pm 0.02$ & $0.097 \pm 0.06$ \\
Prevalence infected mosquitoes (\%) & $82.28(29 / 35)$ & 0.02 \\
Oocytes mean per infected mosquitoes & 128 & $0.71(23 / 35)$ \\
\hline
\end{tabular}

The results are expressed as mean \pm standard errors. The result shown is representative of 1 assay of the 2 independent assays performed

activity too. Indeed, parasitaemia was reduced by $80 \%$ in mice infected with $10^{7} \mathrm{P}$. yoelii infected red blood cells when $\mathrm{MB}(50 \mathrm{mg} / \mathrm{kg})$ was applied from day 0 to day 3 (Table 2), leading to the disappearance of gametocytes from the circulation. These results are in agreement with preliminary clinical data showing a complete elimination of asexual stages and gametocytes in the blood stream of MB-treated malaria patients [18, 39]. Even more interesting, $\mathrm{MB}$ considerably decreased the prevalence of infected mosquitoes when the treatment was administrated $2 \mathrm{~h}$ before feeding. These observations are in line with results on gametocyte development in the P. falciparum model [38].

Why is MB little effective at the hepatic level while it is very active on blood stage and gametocytes? A failure of its uptake into the infected host cell? The typical example is fosmidomycin, an antibiotic with relatively rapid action against asexual blood stages. Previous work established that fosmidomycin is efficiently transported into $P$. falciparum-infected erythrocytes through parasite-induced new permeability pathways. In contrast, Plasmodium berghei liver-stage parasites were shown to be resistant to fosmidomycin, presumably due to the inability of this compound to enter hepatocytes [48-50].
Another explanation could be that, similarly to PQ [8], MB would require metabolization by hepatic cytochromes to become active. This would imply that the effect of MB on Plasmodium blood stages is due to one or more active metabolites. This is the case of several drugs that are considered prodrugs. For instance, proguanil is a prodrug and its cyclisation by cytochrome P450 into pharmacologically active triazine metabolite (cycloguanil) inhibits plasmodial dihydrofolate reductase. This biotransformation is catalyzed by CYP2C19 and many anti-malarial lead compounds are being designed and synthesized to exploit this pathway [51].

\section{Conclusion}

This study confirms that $\mathrm{MB}$, a precursor of $\mathrm{PQ}$, belongs to the large group of anti-malarial compounds active against malaria blood-stage parasites. Moreover it has an effect on gametocytes, thus preventing transmission to mosquitoes. Unfortunately, MB, in the formulation used in endemic areas, is likely inactive against Plasmodium hepatic stage.

\section{Abbreviations}

DAPI: 4',6-Diamidino-2-phénylindole; MB: methylene blue; PQ: primaquine; PVB: Proveblue; G6PD: glucose-6 phosphate dehydrogenase; TI: therapeutic 
index; $\mathrm{IC}_{50}$ : drug concentration that kill $50 \%$ of parasites; $\mathrm{TC}_{50}$ : drug concentration that kill $50 \%$ of the hepatocytes; EEFs: exo-erythrocytic forms.

\section{Authors' contributions}

HBV and JFF conducted all in vitro studies and data analyses. HBV, MT and BK conducted all animal experiments and assisted with data analyses. DM conceived the study, and participated in its design and coordination. OS provided the transgenic $P$. yoelii parasites. OM initiated the study of stage-specific activity of methylene blue. JCV provides pieces of liver for liver perfusion to obtain human hepatocytes. NDB and RLG provide monkey for the production of $P$. cynomolgi sporozoites. HBV, DS, VS, OS and DM wrote the manuscript. All authors contributed to the interpretation of the data. All authors read and approved the final manuscript.

\section{Author details}

1 Sorbonne Université, Inserm, CNRS, Centre d'Immunologie et des Maladies Infectieuses, U1135, ERL8255, CIMI-Paris, F-75013 PARIS, France. ${ }^{2}$ Département de Parasitologie-Mycologie, UFR des Sciences Pharmaceutiques et Biologiques, Université Félix Houphouët Boigny, Abidjan, Côte d'Ivoire. ${ }^{3}$ Department of Dermatology, Eberhard Karls University, Tübingen, Germany. ${ }^{4}$ UPMC, UMS28, 105 Bd de l'hôpital, 75013 Paris, France. ${ }^{5}$ Service de Chirurgie Digestive, Hépato-Bilio-Pancréatique et Transplantation Hépatique, AP-HP, Groupe Hospitalier Pitié-Salpêtrière, 83 Bd de l'hôpital, 75013 Paris, France. ${ }^{6}$ German Center for Infection Research (DZIF), Tübingen, Germany. ${ }^{7}$ Institute for Tropical Medicine, University of Tübingen, Tübingen, Germany. ${ }^{8}$ Institute of Public Health, Medical School, Ruprecht-Karls-University, Heidelberg, Germany. ${ }^{9}$ CEA, INSERM U1184, Immunology of Viral Infections and Autoimmune Diseases, Université Paris Sud 11, Fontenay-aux-Roses, France. ${ }^{10}$ Service de Parasitologie-Mycologie, Centre National de Référence du Paludisme, AP-HP, Groupe Hospitalier Pitié Salpêtrière, 83 Bd de l'hôpital, 75013 PARIS, France.

\section{Acknowledgements}

We thank Provepharm Company (Marseille) for the special provision conditions concerning the MB powder (Proveblue TM 27000760). This work benefited from equipment and services from the CELIS cell culture core facility (Institut du Cerveau et de la Moelle Epinière, Paris), a platform supported through the ANR grants, ANR-10-IAIHU-06 and ANR-11-INBS-0011-NeurATRIS. We are particularly grateful to David Akbar for his valuable assistance regarding automated fluorescence imaging. This work was supported by the "investissements d'avenir" programs managed by the ANR under reference ANR-11-INBS-0008 funding the Infection Diseases Models and Innovative Therapies (IDMIT, Fontenay-aux-Roses, France) Infracstructure, and ANR-10-EQPX-02-01 funding the flowCyTech facility (IDMIT, Fontenay-aux-Roses, France).

\section{Competing interests}

The authors declare that they have no competing interests.

\section{Availability of data and materials section}

The datasets used and analysed during the current study are available from the corresponding author on reasonable request.

\section{Ethics approval and consent to participate}

All procedures involving animal models complied with European regulations. The protocol was ethically approved by the Ministère de l'Education Nationale, de l'Enseignement Supérieur et de la Recherche (Authorization Number 01737.03).

\section{Funding}

This work was supported by an ANR grant'HypEpic' to DM and the French Parasitology consortium grant ParaFrap (ANR-11-LABX0024) to DM and OS. HBV was supported by the SCAC fellowship (French Embassy in Côte d'Ivoire).

\section{Publisher's Note}

Springer Nature remains neutral with regard to jurisdictional claims in published maps and institutional affiliations.

Received: 18 September 2017 Accepted: 26 March 2018

Published online: 03 April 2018

\section{References}

1. White NJ, Pukrittayakamee S, Hien TT, Faiz MA, Mokuolu OA, Dondorp AM. Malaria. Lancet. 2014;383:723-35.

2. WHO. World malaria report 2016. Geneva: World Health Organization; 2016.

3. White NJ. Can new treatment developments combat resistance in malaria? Expert Opin Pharmacother. 2016;17:1303-7.

4. Biamonte MA, Wanner J, Le Roch KG. Recent advances in malaria drug discovery. Bioorg Med Chem Lett. 2013:23:2829-43.

5. Oliveira R, Miranda D, Magalhães J, Capela R, Perry MJ, O'Neill PM, et al. From hybrid compounds to targeted drug delivery in antimalarial therapy. Bioorg Med Chem. 2015;23:5120-30.

6. Burrows JN, van Huijsduijnen RH, Möhrle JJ, Oeuvray C, Wells TNC. Designing the next generation of medicines for malaria control and eradication. Malar J. 2013;12:187.

7. Carmona-Fonseca J, Alvarez G, Maestre A. Methemoglobinemia and adverse events in Plasmodium vivax malaria patients associated with high doses of primaquine treatment. Am J Trop Med Hyg. 2009;80:188-93.

8. Potter BMJ, Xie LH, Vuong C, Zhang J, Zhang P, Duan D, et al. Differential CYP 2 D6 metabolism alters primaquine pharmacokinetics. Antimicrob Agents Chemother. 2015;59:2380-7.

9. Rajapakse S, Rodrigo C, Fernando SD. Tafenoquine for preventing relapse in people with Plasmodium vivax malaria. Cochrane Database Syst Rev. 2015;(4):CD010458.

10. Ebstie YA, Abay SM, Tadesse WT, Ejigu DA. Tafenoquine and its potential in the treatment and relapse prevention of Plasmodium vivax malaria: the evidence to date. Drug Des Devel Ther. 2016;10:2387-99.

11. Vennerstrom JL, Makler MT, Angerhofer CK, Williams JA. Antimalarial dyes revisited: xanthenes, azines, oxazines, and thiazines. Antimicrob Agents Chemother. 1995;39:2671-7.

12. Synthetic Schulemann W, Preparations Anti-Malarial. Proc R Soc Med. 1932;25:897-905

13. Guttmann P, Ehrlich P. Ueber die Wirkung des Methylenblau bei Malaria. Berlin Klin. Wochenschr. 1960;28:953-6.

14. Schirmer RH, Adler H, Pickhardt M, Mandelkow E. Lest we forget you methylene blue. Neurobiol Aging. 2011;32:e7-16.

15. Schirmer RH, Coulibaly B, Stich A, Scheiwein M, Merkle H, Eubel J, et al. Methylene blue as an antimalarial agent. Redox Rep Commun Free Radic Res. 2003;8:272-5.

16. Pascual A, Henry M, Briolant S, Charras S, Baret E, Amalvict R, et al. In vitro activity of Proveblue (methylene blue) on Plasmodium falciparum strains resistant to standard antimalarial drugs. Antimicrob Agents Chemother. 2011;55:2472-4.

17. Meissner PE, Mandi G, Witte S, Coulibaly B, Mansmann U, Rengelshausen $J$, et al. Safety of the methylene blue plus chloroquine combination in the treatment of uncomplicated falciparum malaria in young children of Burkina Faso. Malar J. 2005;4:45.

18. Coulibaly B, Pritsch M, Bountogo M, Meissner PE, Nebié E, Klose C, et al. Efficacy and safety of triple combination therapy with artesunate-amodiaquine-methylene blue for falciparum malaria in children: a randomized controlled trial in Burkina Faso. J Infect Dis. 2015;211:689-97.

19. Müller O, Mockenhaupt FP, Marks B, Meissner P, Coulibaly B, Kuhnert R, et al. Haemolysis risk in methylene blue treatment of G6PD-sufficient and G6PD-deficient West-African children with uncomplicated falciparum malaria: a synopsis of four RCTs. Pharmacoepidemiol Drug Saf. 2013;22:376-85.

20. Manzoni G, Briquet S, Risco-Castillo V, Gaultier C, Topçu S, Ivănescu ML, et al. A rapid and robust selection procedure for generating drug-selectable marker-free recombinant malaria parasites. Sci Rep. 2014;4:4760.

21. Silvie $O$, Rubinstein E, Franetich J-F, Prenant M, Belnoue E, Rénia L, et al. Hepatocyte CD81 is required for Plasmodium falciparum and Plasmodium yoelii sporozoite infectivity. Nat Med. 2003;9:93-6.

22. Dembele L, Gego A, Zeeman A-M, Franetich J-F, Silvie O, Rametti A, et al. Towards an in vitro model of plasmodium hypnozoites suitable for drug discovery. PLoS ONE. 2011;6:e18162.

23. Rénia L, Mattei D, Goma J, Pied S, Dubois P, Miltgen F, et al. A malaria heatshock-like determinant expressed on the infected hepatocyte surface is the target of antibody-dependent cell-mediated cytotoxic mechanisms by nonparenchymal liver cells. Eur J Immunol. 1990;20:1445-9. 
24. Le Nagard H, Vincent C, Mentré F, Le Bras J. Online analysis of in vitro resistance to antimalarial drugs through nonlinear regression. Comput Methods Programs Biomed. 2011;104:10-8.

25. Mwakingwe A, Ting L-M, Hochman S, Chen J, Sinnis P, Kim K. Noninvasive real-time monitoring of liver-stage development of bioluminescent Plasmodium parasites. J Infect Dis. 2009;200:1470-8.

26. Matos J, da Cruz FP, Cabrita É, Gut J, Nogueira F, do Rosário VE, et al. Novel potent metallocenes against liver stage malaria. Antimicrob Agents Chemother. 2012;56:1564-70.

27. Delves M, Plouffe D, Scheurer C, Meister S, Wittlin S, Winzeler EA, et al. The activities of current antimalarial drugs on the life cycle stages of Plasmodium: a comparative study with human and rodent parasites. PLoS Med. 2012;9:e1001169.

28. Garavito G, Bertani S, Rincon J, Maurel S, Monje MC, Landau I, et al. Blood schizontocidal activity of methylene blue in combination with antimalarials against Plasmodium falciparum. Parasite. 2007;14:135-40.

29. Dormoi J, Pascual A, Briolant S, Amalvict R, Charras S, Baret E, et al. Proveblue (methylene blue) as an antimalarial agent: in vitro synergy with dihydroartemisinin and atorvastatin. Antimicrob Agents Chemother. 2012;56:3467-9.

30. Fall B, Camara C, Fall M, Nakoulima A, Dionne P, Diatta B, et al. Plasmodium falciparum susceptibility to standard and potential anti-malarial drugs in Dakar, Senegal, during the 2013-2014 malaria season. Malar J. 2015:14:60.

31. Dormoi J, Briolant S, Desgrouas C, Pradines B. Efficacy of proveblue (methylene blue) in an experimental cerebral malaria murine model. Antimicrob Agents Chemother. 2013;57:3412-4.

32. Dormoi J, Pradines B. Dose responses of proveblue methylene blue in an experimental murine cerebral malaria model. Antimicrob Agents Chemother. 2013;57:4080-1.

33. Dormoi J, Briolant S, Desgrouas C, Pradines B. Impact of methylene blue and atorvastatin combination therapy on the apparition of cerebral malaria in a murine model. Malar J. 2013;12:127.

34. Mandi G, Witte S, Meissner P, Coulibaly B, Mansmann U, Rengelshausen $J$, et al. Safety of the combination of chloroquine and methylene blue in healthy adult men with G6PD deficiency from rural Burkina Faso. Trop Med Int Health. 2005;10:32-8.

35. Meissner PE, Mandi G, Coulibaly B, Witte S, Tapsoba T, Mansmann U, et al. Methylene blue for malaria in Africa: results from a dose-finding study in combination with chloroquine. Malar J. 2006;5:84.

36. Zoungrana A, Coulibaly B, Sié A, Walter-Sack I, Mockenhaupt FP, Kouyaté $B$, et al. Safety and efficacy of methylene blue combined with artesunate or amodiaquine for uncomplicated falciparum malaria: a randomized controlled trial from Burkina Faso. PLoS ONE. 2008:3:e1630.

37. Bountogo M, Zoungrana A, Coulibaly B, Klose C, Mansmann U, Mockenhaupt FP, et al. Efficacy of methylene blue monotherapy in semi-immune adults with uncomplicated falciparum malaria: a controlled trial in Burkina Faso. Trop Med Int Health. 2010;15:713-7.

38. Adjalley SH, Johnston GL, Li T, Eastman RT, Ekland EH, Eappen AG, et al. Quantitative assessment of Plasmodium falciparum sexual development reveals potent transmission-blocking activity by methylene blue. Proc Natl Acad Sci USA. 2011;108:E1214-23.
39. Coulibaly B, Zoungrana A, Mockenhaupt FP, Schirmer RH, Klose C, Mansmann $U$, et al. Strong gametocytocidal effect of methylene bluebased combination therapy against falciparum malaria: a randomised controlled trial. PLoS ONE. 2009;4:e5318.

40. Schmidt LH. Compatibility of relapse patterns of Plasmodium cynomolgi infections in rhesus monkeys with continuous cyclical development and hypnozoite concepts of relapse. Am J Trop Med Hyg. 1986:35:1077-99.

41. Deye GA, Gettayacamin M, Hansukjariya P, Im-erbsin R, Sattabongkot J, Rothstein Y, et al. Use of a rhesus Plasmodium cynomolgi model to screen for anti-hypnozoite activity of pharmaceutical substances. Am J Trop Med Hyg. 2012;86:931-5

42. Putrianti ED, Silvie O, Kordes M, Borrmann S, Matuschewski K. Vaccine-like immunity against malaria by repeated causal-prophylactic treatment of liver-stage Plasmodium parasites. J Infect Dis. 2009;199:899-903.

43. Friesen J, Silvie O, Putrianti ED, Hafalla JCR, Matuschewski K, Borrmann S. Natural immunization against malaria: causal prophylaxis with antibiotics. Sci Transl Med. 2010;2:40ra49.

44. Ashley EA, Recht J, White NJ. Primaquine: the risks and the benefits. Malar J. 2014;13:418.

45. Annoura T, Chevalley S, Janse CJ, Franke-Fayard B, Khan SM. Quantitative analysis of Plasmodium berghei liver stages by bioluminescence imaging. Methods Mol Biol. 2013;923:429-43.

46. Miller JL, Murray S, Vaughan AM, Harupa A, Sack B, Baldwin M, et al. Quantitative bioluminescent imaging of pre-erythrocytic malaria parasite infection using luciferase-expressing Plasmodium yoelii. PLoS ONE. 2013;8:e60820.

47. Garavito G. Etude pharmacologique expérimentale de l'activité antipaludique d'un composé de synthèse: le chlorure de methyl-thioninium. PhD Thesis, Université Toulouse III/Paul Sabatier École Doctorale BSB, IOP Publishing Physics, Toulouse, 2007; pp 155.

48. Sparr C, Purkayastha N, Kolesinska B, Gengenbacher M, Amulic B, Matuschewski K, et al. Improved efficacy of fosmidomycin against Plasmodium and Mycobacterium species by combination with the cell-penetrating peptide octaarginine. Antimicrob Agents Chemother. 2013;57:4689-98.

49. Baumeister S, Wiesner J, Reichenberg A, Hintz M, Bietz S, Harb OS, et al. Fosmidomycin uptake into Plasmodium and Babesia-infected erythrocytes is facilitated by parasite-induced new permeability pathways. PLoS ONE. 2011;6:e19334.

50. Nair SC, Brooks CF, Goodman CD, Sturm A, Strurm A, McFadden Gl, et al. Apicoplast isoprenoid precursor synthesis and the molecular basis of fosmidomycin resistance in Toxoplasma gondii. J Exp Med. 2011;208:1547-59.

51. Arfeen M, Patel DS, Abbat S, Taxak N, Bharatam PV. Importance of cytochromes in cyclization reactions: quantum chemical study on a model reaction of proguanil to cycloguanil. J Comput Chem. 2014:35:2047-55.

52. Carraz M, Jossang A, Franetich J-F, Siau A, Ciceron L, Hannoun L, et al. A plant-derived morphinan as a novel lead compound active against malaria liver stages. PLoS Med. 2006;3:e513.

\section{Submit your next manuscript to BioMed Central and we will help you at every step:}

- We accept pre-submission inquiries

- Our selector tool helps you to find the most relevant journal

- We provide round the clock customer support

- Convenient online submission

- Thorough peer review

- Inclusion in PubMed and all major indexing services

- Maximum visibility for your research

Submit your manuscript at www.biomedcentral.com/submit 